\title{
Carrier screening for (CGG)n repeat expansion of FMR1 gene in Korean women
}

\author{
Kyung Min Kang ${ }^{1}$, Se Ra Sung ${ }^{1}$, Ji Eun Park', Yun Jeong Shin, Sang Hee Park¹, Mi Uk Chin ${ }^{1}$, Sang Woo Lyu, \\ Dong Hyun Cha, ${ }^{1,2}$, and Sung Han Shim ${ }^{1,3, *}$ \\ ${ }^{1}$ Genetics Laboratory, Fertility Center of CHA Gangnam Medical Center, Seoul, Korea \\ ${ }^{2}$ Department of Obstetrics and Gynecology, CHA Gangnam Medical Center, Seoul, Korea \\ ${ }^{3}$ Department of Biomedical Science, College of Life Science, CHA University, Seoul, Korea
}

Purpose: We examined the prevalence and CGG/AGG repeat structure of expanded alleles of the FMR1 gene in preconceptional and pregnant Korean women.

Materials and Methods: The CGG repeats in the FMR1 genes of 1,408 women were analyzed by polymerase chain reaction and Southern blot analysis. To estimate the prevalence of expansion alleles, the individuals were divided into low risk and high risk group.

Results: Within this population, $98.4 \%$ had normal alleles and $1.6 \%$ had abnormal alleles including intermediate $(0.6 \%)$, premutation $(0.5 \%)$, full mutation $(0.1 \%)$, and hemizygous $(0.4 \%)$ alleles. There were 2 premutation alleles $(1: 666,95 \%$ confidence interval [CI] 1:250-1,776) in the low risk group and 5 premutation alleles (1:15, 95\% 1:6-36) in the high risk group. There were 8 intermediate alleles $(1: 167,95 \% \mathrm{Cl}$ 1:130-213) in the low risk group and 1 intermediate alleles $(1: 76,95 \% \mathrm{Cl}$ 1:11-533) in the high group. Six of the 7 premutation alleles did not contain AGG interruptions within the repeats and 1 had a single AGG interruption. Four of the 9 intermediate alleles contained 2-3 AGG, 4 had a single AGG, and 1 had no AGG interruptions.

Conclusion: Our study demonstrates the prevalence and CGG/AGG structure of expansion alleles in Korean women. The identified premutation prevalence is higher than that of other Asian populations and lower than that of Caucasian populations. Although our study is limited by size and population bias, our findings could prove useful for genetic counseling of preconceptional or pregnant women.

Key words: Fragile X syndrome, Trinucleotide repeat expansion, Primary ovarian failure, Gene frequency, Carrier state.

\section{Introduction}

Fragile X syndrome (FXS) is one of the most common mental retardation diseases and a major risk factor of inherited intellectual disabilities. FXS is caused by an expansion of CGG repeats in the FMR1 gene, which are hypermethylated within $\mathrm{CpG}$ islands at the promoter region, leading to transcriptional silencing of the FMR 1 gene and the absence of fragile $\mathrm{X}$ mental

Received: 1 June 2016, Revised: 17 June 2016, Accepted: 17 June 2016, Published: 30 June 2016

${ }^{*}$ Corresponding author: Sung Han Shim, Ph.D.

Genetics Laboratory, Fertility Center of CHA Gangnam Medical Center, CHA University, 566 Nonhyon-ro, Gangnam-gu, Seoul 06135, Korea.

Tel: +82-2-3453-6438, Fax: +82-2-3453-6450, E-mail: shshim@cha.ac.kr

Conflict of interest: The authors declare that they do not have any conflicts of interest.

(c) This is an open-access article distributed under the terms of the Creative Commons Attribution Non-Commercial License (http://creativecommons.org/licenses/by-nc/4.0/) which permits unrestricted non-commercial use, distribution, and reproduction in any medium, provided the original work is properly cited.

(c) Copyright 2016 by the Korean Society of Medical Genetics 
retardation protein translation [1].

According to the American College of Medical Genetics and Genomics guidelines, expanded alleles can be classified as follows; normal (6-44 CGG repeats), intermediate or gray zone (GZ; 45-54 CGG repeats), premutation (55-200 repeats), or full mutation ( $>200$ repeats). Most normal individuals have $6-44$ CGG repeats including 2-3 interrupting AGG trinucleotides, found at positions 10 or 11, and 20 or 21 of the CGG repeat tract $[2,3]$. Premutation carriers have 55-200 CGG repeats with 1-2 $A G G$ interruptions or tend to have fewer $A G G$ interruptions resulting in an increasing length of the $C G G$ repeat tract. The loss of AGG interruption appears to increase repeat instability and the risk of full mutation expansion during transmission of maternal premutation alleles $[2,4]$. Intermediate or $\mathrm{GZ}$ alleles (45-54 repeats) are potentially unstable and thus their clinical implications are currently under investigation [5]. Nolin et al. [5] defined the role of the AGG interruptions in the instability of 45-54 repeat alleles and identified a 19-fold difference in alleles with no AGG interruptions compared to those with 2 AGG interruptions. In addition, they found that full mutation expansions were transmitted from alleles with no $A G G$ interruptions.

The aim of this study was to determine the prevalence and CGG/AGG structure of expanded alleles as well as the distribution of the FMR1 alleles in Korean women.

\section{Materials and Methods}

\section{Subjects}

A total of 1,408 preconceptional or pregnant women were tested for fragile X carrier screening at CHA Gangnam Medical Center (Seoul, Korea) between March 2010 and December 2015. The individuals applied for testing on their own initiative or on the advice of their physician, on a self-pay basis. Each of the women completed a questionnaire to ascertain any history of FXS, mental retardation, or premature ovarian failure (POF). All women identified as carrier with a premutation allele were offered genetic counseling. Those women who were already pregnant and preconceptional women who became pregnant were provided with information regarding prenatal diagnosis by amniotic-fluid analysis or chorionic villus sampling. This study was approved by the Institutional Review Board of Cha Gangnam Medical Center (IRB No. GCl-13-35).

\section{DNA extraction and FMR1 region-specific CGG PCR}

Genomic DNA was extracted from peripheral blood samples using the QuickGene DNA kit (Fuijifilm Co., Tokyo, Japan) according to the manufacturer's instructions. The (CGG)n repeats of the FMR 1 gene were analyzed by polymerase chain reaction (PCR) using the GC rich PCR system kit (Roche Diagnostics, Mannheim, Germany).

\section{FMR1 triplet repeat-primed (TP)-PCR and genetic analyzer}

To confirm the number and structure of CGG/AGG repeats in intermediate, premutation and full mutation samples, we used the AmplideX FMR1 kit (Asuragen Inc., Austin, TX, USA) according to the manufacturer's instructions. The size of the PCR products was converted into the number of repeats using an Microsoft Excel-based data analysis macro (FMR 1 analysis macro version 2.1.2; Asuragen Inc.). A mixed internal standard DNA sample was tested in the same plate for each experiment to provide a process control $[5,6]$. The PCR products were loaded on an ABI Prism 3130xI Genetic Analyzer (Applied Biosystems, Foster City, CA, USA) and analyzed with GeneMapper version 3.2 (Applied Biosystems); $1 \mu \mathrm{L}$ of the PCR products mixed with $9 \mu \mathrm{L}$ formamide (Applied Biosystems) and $0.3 \mu \mathrm{L}$ GeneScan $^{\mathrm{TM}}$ -500LIZ ${ }^{\mathrm{TM}}$ internal size standard (Applied Biosystems) internal size standard were loaded into the genetic analyzer.

\section{Southern blot analysis}

Southern blots were performed to identify premutation or full mutation when the PCR results were inconclusive (only one allele within normal range of [CGG]n repeats or amplification failed). Genomic DNA (10 $\mu \mathrm{g})$ was digested with EcoRI and Eagl (New England Biolabs, Ipswich, MA, USA). Hybridization and detection were performed using a DIG-labeled PCR probe (forward; TGAAGAGAAGATGGAGGAGCT/reverse; TCTCATTCGATAGGCGCTAG) according to Gold et al. [7].

\section{Results}

Of 1,408 individuals 1,385 samples (98.4\%) were within the

Table 1. Number of FMR1 triplet repeats

\begin{tabular}{|cc|}
\hline \multicolumn{1}{c}{ CGG repeat range } & \multicolumn{1}{c}{ Sample } \\
\hline $5-45$ (normal) & $1,385(98.4)$ \\
\hline $46-54$ (intermediate) & $9(0.6)$ \\
\hline $55-200$ (premutation) & $7(0.5)$ \\
\hline 200 (full mutation) & $1(0.1)$ \\
\hline Only one normal allele (hemizygote) & $6(0.4)$ \\
\hline Total & $1,408(100)$ \\
\hline
\end{tabular}

Values are presented as number (\%). 
normal range, 2 normal CGG repeat (16-44 repeats) alleles. There were 17 samples with one normal allele and one expanded allele; 9 had an intermediate (45-54 repeats) allele (0.6\%), 7 had a premutation (55-200 repeats) allele (0.5\%) and 1 had a full mutation ( $>200$ repeats) allele $(0.1 \%)$. The remaining 6 samples $(0.4 \%)$ were hemizygous, meaning they had only a single normal X chromosome or one normal and a partially deleted X chromosome (Table 1).

The most prevalent alleles were 29 (45.0\%), 30 (31.3\%) and 36 repeats $(10.1 \%)$ and these three alleles accounted for $86.4 \%$ of the total (Fig. 1).

The allele patterns identified in this study was shown in Fig. 2. Nine hundreds and eighteen samples (65.2\%) were heterozygous and 490 (34.8\%) were homozygous. Of the heterozygous samples, 903 had two normal alleles, 9 (1.0\%) had one normal allele and one intermediate allele and 6 were premutation carriers $(0.7 \%)$. For homozygous samples, Southern blot analyses were performed to identify whether the homozygous samples had a large expansion allele (premutation or full mutation). One case (0.2\%) was identified as a premutation carrier and one case $(0.2 \%)$ had a full mutation allele. Interestingly, 6 cases (1.2\%) were hemizygous. The CGG repeat length in these premutation, full mutation and hemizygous samples was then re-examined using TP-PCR. The premutation sample was shown to contain 29/93 repeats and the full mutation sample had $>200$ repeats (Fig. 3A). The hemizygous samples were shown to contain only the $2.8 \mathrm{~kb}$ unmethylated X chromosome allele; the $5.2 \mathrm{~kb}$ methylated allele

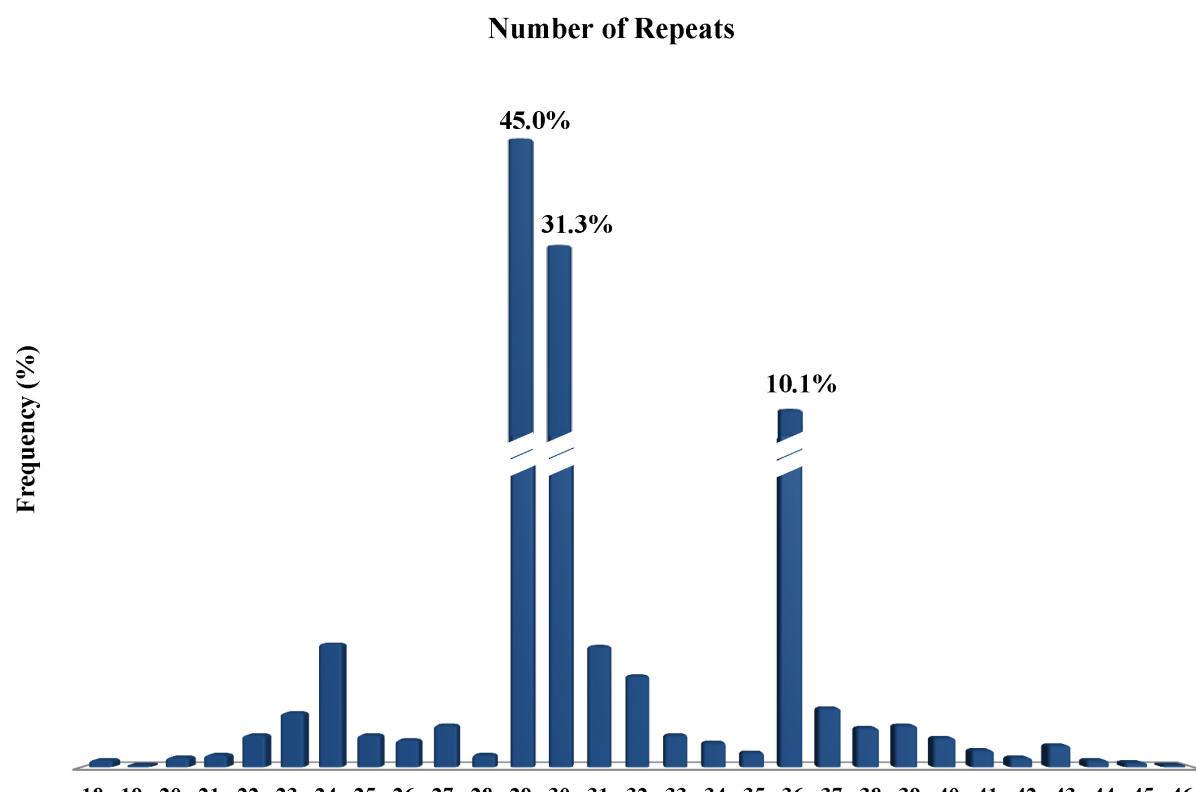

$\begin{array}{lllllllllllllllllllllllllllll}18 & 19 & 20 & 21 & 22 & 23 & 24 & 25 & 26 & 27 & 28 & 29 & 30 & 31 & 32 & 33 & 34 & 35 & 36 & 37 & 38 & 39 & 40 & 41 & 42 & 43 & 44 & 45 & 46\end{array}$
Fig. 1. Distribution of triplet repeats in normal FMR1 alleles.

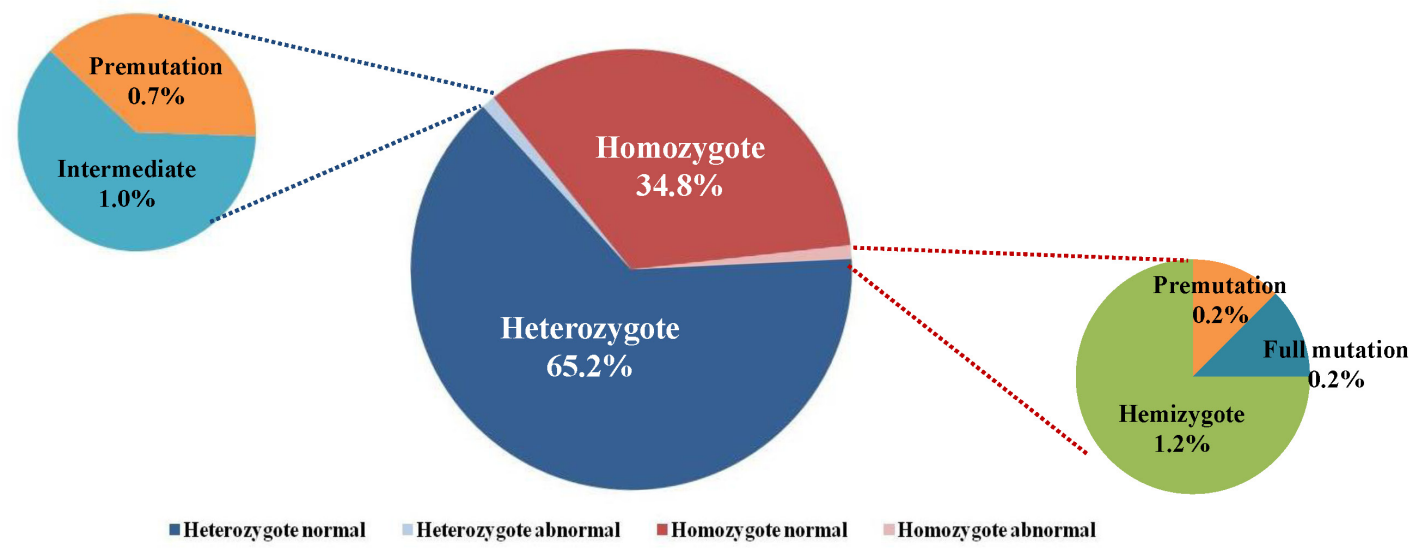

Fig. 2. The results for alleles pattern of FMR1 gene in total samples. 
A.

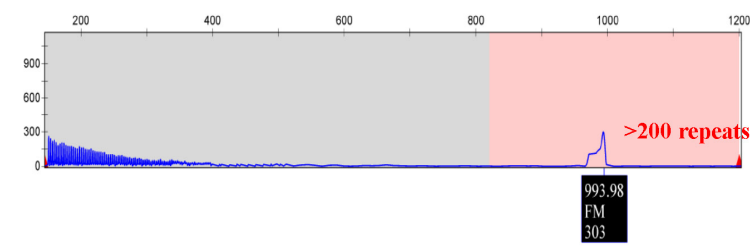

b
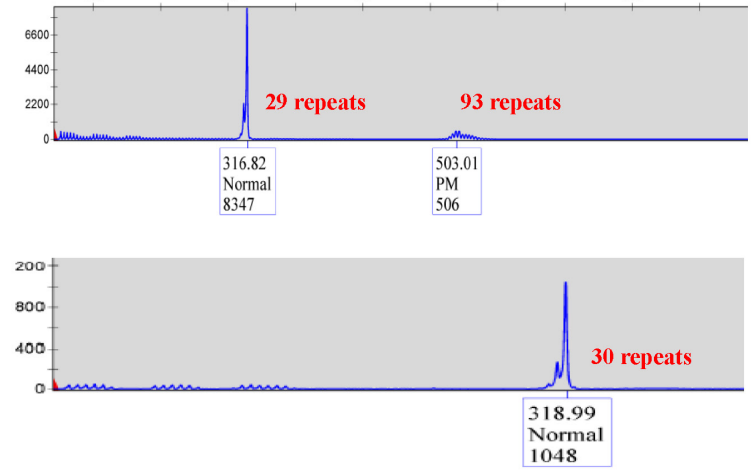

B.

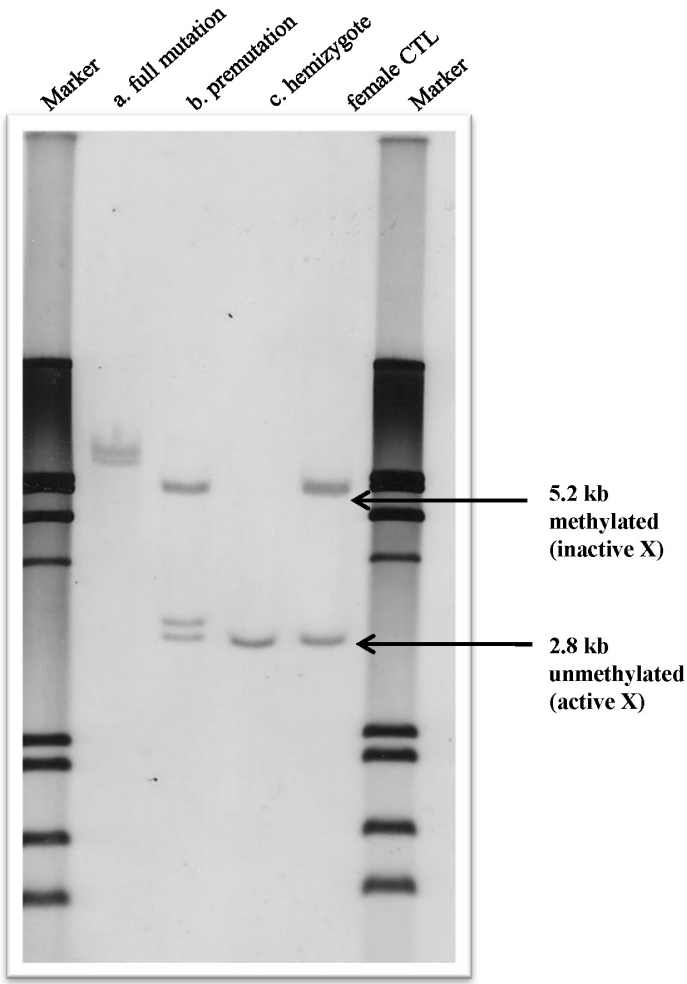

Fig. 3. Characterization of homozygous FMR1 samples. (A) Analysis of CGG repeat number; a, >200; b, 29 and 93 repeats; and c, 30 repeats (hemizygous). (B) Southern blot analysis.

was not detected in these samples (Fig. 3B).

The CGG/AGG interspersion patterns were examined in 17 samples with an expanded allele (intermediate, premutation, and full mutation allele). Six of the 7 premutation alleles did not contain $A G G$ interruptions within the repeats and 1 had a single $A G G$ interruption (data not shown). In contrast, 4 of the 9 intermediate alleles contained 2-3 AGG interruption, 4 had a single AGG with an expanded 3' pure CGG repeat stretch (4143 repeats) and 1 had no $A G G$ interruptions. Intermediate range alleles with 2-3 AGG interruptions exhibited a similar pattern to that of normal alleles, while alleles with 0-1 AGG interruptions demonstrated a similar pattern to that of premutation alleles with 0-1 AGG (Table 2).

\section{Discussion}

In this study, we screened 1,408 Korean women for (CGG) $\mathrm{n}$ repeat expansion of FMR 1 gene. Of total samples, abnormal samples containing intermediate, premutation, full mutation and hemizygous alleles accounted for $1.6 \%(n=23)$. The distribution of normal FMR1 alleles was 29, 30 and 36 repeats (86.4\%), similar to that of other Korean population $[8,9]$.

For the more details, the samples were divided into two
Table 2. CGG/AGG repeat structure of the FMR1 gene in women with intermediate alleles

\begin{tabular}{clc}
$\begin{array}{c}\text { Expanded } \\
\text { allele }\end{array}$ & \multicolumn{1}{c}{ CGG repeat structure } & AGG (n) \\
\hline 48 & (CGG10)AGG(CGG9)AGG(CGG10)AGG(CGG16) & 3 \\
49 & (CGG9)AGG(CGG9)AGG(CGG19) AGG(CGG9) & 3 \\
49 & (CGG9)AGG(CGG9)AGG(CGG19) AGG(CGG9) & 3 \\
51 & (CGG9)AGG(CGG41) & 1 \\
52 & (CGG9)AGG(CGG42) & 1 \\
52 & (CGG10)AGG(CGG41) & 1 \\
53 & (CGG10)AGG(CGG24)AGG(CGG17) & 2 \\
53 & (CGG53) & 0 \\
54 & (CGG10)AGG(CGG43) & 1 \\
\hline
\end{tabular}

groups a low risk group without indication of FXS and a high risk group with an indication of FXS history, mental retardation or gynecological diseases such as POF. we identified 8 intermediate alleles in the low risk group (1:167, 95\% Cl 1:130-213), 1 intermediate allele in the high risk group $(1: 76,95 \% \mathrm{Cl} 1: 11-$ 533), 2 premutation alleles in the low risk group $(1: 666,95 \% \mathrm{Cl}$ $1: 250-1,776), 5$ premutation alleles in the high risk group $(1: 15$, 95\% Cl 1:6-36), 1 full mutation allele in the high risk group (1:76, 95\% Cl 1:11-533), 1 hemizygous allele in the low risk group (1:1332,95\% $\mathrm{Cl} 1: 188-9456)$ and 5 hemizygous alleles in the 
Table 3. Cytogenetic analyses of hemizygous FMR1 samples

\begin{tabular}{|c|c|c|c|}
\hline No. & Age (yr) & Indication & Cytogenetic analysis \\
\hline 1 & 26 & POF & $46, X X, \operatorname{dup}(X p), \operatorname{del}(X q)$ \\
\hline 2 & 32 & POF & $46, X, \operatorname{del}(X)(q 26)$ \\
\hline 3 & 28 & POF & $46, X, \operatorname{del}(X)(q 21.3)$ \\
\hline 4 & 18 & POF & $46, X, \operatorname{del}(X)(q 21.2)$ \\
\hline 5 & 29 & Sterility & mos 46,X,psu idic(X)(q22.3)[81]/45,X[18]/46,XX[1] \\
\hline 6 & Fetus & Screening & 46,X,der(X).ish $\operatorname{der}(X) t(X ; Y)(q 28 ; q 11.23)(S R Y-, D Y Z 3-, D Y Z 1+, t e l X q Y q+)$ \\
\hline
\end{tabular}

high risk group (1:15,95\% $\mathrm{Cl}$ 1:6-36). As mentioned just earlier, the prevalence of premutation carriers in our study was 1:666, similar to Kim et al. (1:781) in Korean population [8]. This finding is higher the prevalence of other Asian populations and lower than that of Caucasian populations $[8,9]$. Our population-based screening for FXS may not directly reflect the overall prevalence in Korean women because the study was limited to women who requested the test themselves or were referred by a doctor and had to pay for it.

Of the 6 hemizygous samples, 4 had an indication of POF, 1 was infertile for unknown reasons and 1 was detected from the prenatal test of a pregnant woman. Table 3 details the cytogenetic analyses of the hemizygous samples. The 6 hemizygous samples contained the $2.8 \mathrm{~kb}$ unmethylated allele of the active $X$ chromosome and 4 of the hemizygous samples had an indication of POF. In 3-15\% of women premutation aberrations of the FMR 1 gene can manifest as a POF/POI phenotype [10]; $10-15 \%$ of the cases are $X$ chromosome abnormalities, such as numerical and structural aberrations (deletions, inversions, and X/autosome translocations) $[11,12]$. Haploinsufficiency of genes located in the missing region of the $\mathrm{X}$ chromosome could provide a promising explanation for the POF disease background, especially when it involves $\mathrm{Xq28}$. A haploinsufficient gene is defined as a gene that requires 2 functional alleles for wild type expression. Lack of gene expression because of $\mathrm{X}$ inactivation may affect ovarian function [10].

Inherited patterns of three cases with a premutation allele were examined. The premutation alleles of patient I (29/129 repeats) was transmitted to her daughter and underwent expansion to full mutation. Patient II, a pregnant woman with premutation alleles, has a son with FXS and full mutation alleles were detected in her prenatal testing. Patient III was found to have premutation alleles (29/93); the sister of patient III also has premutation alleles, however, her alleles are further expanded (29/96; data not shown).

Our results revealed dynamic mutation events in the (CGG) $\mathrm{n}$ repeat structure. One patient with intermediate allele (29/53 repeats, no AGG interruptions) had the POF disease background (Anti-Müllerian hormone: >0.14). Bodega et al. [13] demonstrated a significant prevalence of intermediate alleles in the POF population with respect to control group. Rybak et al. [14] reported that sisters with POF had intermediate alleles (45 repeats). In contrast, in Bennett et al. [15] and Guo et al. [16], they showed that the prevalence of intermediate FMR 1 was not increased significantly in sporadic POF than that in controls. So, further study should be performed about association of intermediate allele with POF.

Although an allele is within the intermediate range, if it does not contain AGG interruptions or the length of the 3' pure CGG repeat is greater than the appropriate level, the instability of the CGG repeat region might be increased and it could then expand into a premutation allele $[5,6]$. Thus, it is important that this information be conveyed not only to women with premutation alleles but also to those with intermediate alleles.

Since the identification of the FMR 1 gene in the 1990s, expansion risk estimates have been based solely on maternal repeat length alone. However, data pointing to the role of interspersed AGGs in the stability of the CGG repeat region has accumulated over the past 20 years [17-19]. Nolin et al. $[5,20]$ demonstrated a 19 -fold difference for alleles with no AGGs compared to those with $2 \mathrm{AGG}$ disruptions. Yrigollen et al. [18] showed that the combination of total CGG repeat tract length and the number of AGG interruptions was significantly associated with the risk of expansion to a full mutation. It is important that this more accurate risk information be provided to families through genetic counseling, which will serve to further facilitate their decision making process. Additional studies with larger cohorts and various populations will be required to confirm our findings because these data are limited by study size and population bias. Studies involving the children of patients with intermediate alleles containing 0-1 AGG interruptions might also provide more accurate risk information. Although our study is limited by size and population bias, our 
findings will be useful in terms of genetic counseling offered to preconceptional or pregnant women.

\section{Acknowledgements}

This work was supported by Priority Research Centers Program through the National Research Foundation of Korea (NRF) funded by the Ministry of Education (NRF-2009-0093821).

\section{References}

1. Peprah E. Fragile $X$ syndrome: the FMR1 CGG repeat distribution among world populations. Ann Hum Genet 2012;76:178-91.

2. Eichler EE, Holden JJ, Popovich BW, Reiss AL, Snow K, Thibodeau SN, et al. Length of uninterrupted CGG repeats determines instability in the FMR1 gene. Nat Genet 1994;8:88-94.

3. Kunst $C B$, Warren $S T$. Cryptic and polar variation of the fragile $X$ repeat could result in predisposing normal alleles. Cell 1994;77:85361.

4. Eichler EE, Hammond HA, Macpherson JN, Ward PA, Nelson DL. Population survey of the human FMR1 CGG repeat substructure suggests biased polarity for the loss of AGG interruptions. Hum Mol Genet 1995;4:2199-208.

5. Nolin SL, Sah S, Glicksman A, Sherman SL, Allen E, Berry-Kravis E, et al. Fragile $X$ AGG analysis provides new risk predictions for 45-69 repeat alleles. Am J Med Genet A 2013;161A:771-8.

6. Nolin SL, Glicksman A, Ersalesi N, Dobkin C, Brown WT, Cao R, et al. Fragile $X$ full mutation expansions are inhibited by one or more AGG interruptions in premutation carriers. Genet Med 2015;17:358-64.

7. Gold B, Radu D, Balanko A, Chiang CS. Diagnosis of fragile $X$ syndrome by Southern blot hybridization using a chemiluminescent probe: a laboratory protocol. Mol Diagn 2000;5:169-78.

8. Kim MJ, Kim do J, Kim SY, Yang JH, Kim MH, Lee SW, et al. Fragile X carrier screening in Korean women of reproductive age. J Med Screen 2013;20:15-20.

9. Jang JH, Lee K, Cho EH, Lee EH, Kim JW, Ki CS. Frequency of FMR1 premutation carriers and rate of expansion to full mutation in a retrospective diagnostic FMR1 Korean sample. Clin Genet 2014;85:
441-5.

10. Beke A, Piko H, Haltrich I, Csomor J, Matolcsy A, Fekete G, et al. Molecular cytogenetic analysis of $\mathrm{Xq}$ critical regions in premature ovarian failure. Mol Cytogenet 2013;6:62.

11. Woad KJ, Watkins WJ, Prendergast D, Shelling AN. The genetic basis of premature ovarian failure. Aust N Z J Obstet Gynaecol 2006;46: 242-4.

12. Baronchelli $S$, Conconi D, Panzeri E, Bentivegna A, Redaelli S, Lissoni S, et al. Cytogenetics of premature ovarian failure: an investigation on 269 affected women. J Biomed Biotechnol 2011;2011:370195.

13. Bodega $B$, Bione $S$, Dalprà $L$, Toniolo $D$, Ornaghi $F$, Vegetti $W$, et al. Influence of intermediate and uninterrupted FMR1 CGG expansions in premature ovarian failure manifestation. Hum Reprod 2006;21: 952-7.

14. Rybak EA, Bevilacqua K, Veit CR, Klugman SD, Santoro N. Sibling and self ovum donation for sisters with an intermediate FMR1 mutation: what's a program to do? Fertil Steril 2009;92:394.e9-12.

15. Bennett CE, Conway GS, Macpherson JN, Jacobs PA, Murray A. Intermediate sized CGG repeats are not a common cause of idiopathic premature ovarian failure. Hum Reprod 2010;25:1335-8.

16. Guo $T$, Qin $Y$, Jiao $X$, Li G, Simpson JL, Chen ZJ. FMR1 premutation is an uncommon explanation for premature ovarian failure in Han Chinese. PLoS One 2014;9:e103316.

17. Toledano-Alhadef $H$, Basel-Vanagaite L, Magal N, Davidov B, Ehrlich $S$, Drasinover $V$, et al. Fragile- $X$ carrier screening and the prevalence of premutation and full-mutation carriers in Israel. Am J Hum Genet 2001;69:351-60.

18. Yrigollen CM, Durbin-Johnson $B$, Gane $L$, Nelson DL, Hagerman $R$, Hagerman PJ, et al. AGG interruptions within the maternal FMR1 gene reduce the risk of offspring with fragile $X$ syndrome. Genet Med 2012;14:729-36.

19. Wittenberger MD, Hagerman RJ, Sherman SL, McConkie-Rosell $A$, Welt CK, Rebar RW, et al. The FMR1 premutation and reproduction. Fertil Steril 2007;87:456-65.

20. Nolin SL, Brown WT, Glicksman A, Houck GE Jr, Gargano AD, Sullivan $A$, et al. Expansion of the fragile $X$ CGG repeat in females with premutation or intermediate alleles. Am J Hum Genet 2003;72:45464. 